\title{
MICROFLUIDIC OPTOMECHANICAL OSCILLATORS
}

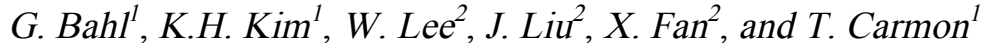

${ }^{1}$ EECS and ${ }^{2}$ BME departments, University of Michigan, Ann Arbor, Michigan, USA

\section{ABSTRACT}

We demonstrate a new class of fused silica MEMS that bridge between optomechanical and fluidic microsystems. Since fused silica is non-conductive and optically transparent, we employ "touch-less" excitation by means of optical electrostriction and radiation pressure to excite surface-acoustic-wave whispering gallery modes and wineglass modes in these devices. This is the first demonstration of mechanical vibration that interacts with fluids, excited in a microstructure via optical forces. These mechanical oscillators operate over several orders-of-magnitude in frequency from $8.5 \mathrm{MHz}-11 \mathrm{GHz}$.

\section{INTRODUCTION}

Resonant MEMS cantilevers with embedded microfluidic channels provide sub-femtogram resolution for mass sensing of analytes in fluidic media [1] using electrostatic transduction. Meanwhile, optical microresonators have proven their use in nanoparticle detection experiments in both wet [2] and dry environments $[3,4]$. Additionally, it is known that light can also be used to excite [5-7] and attenuate [8] mechanical vibration in microdevices by exploiting radiation pressure [5,7], gradient force [9], and electrostriction [6,8]. The excitation of mechanical vibration using optical forces in fluidic media, however, has not been achieved due to the large viscous damping experienced. In this work, we enable this capability by confining the test liquid (distilled water) inside a hollow microfluidic optomechanical $(\mu \mathrm{FOM})$ resonator. We show, for the first time, a device that in the presence of a liquid preserves ultra-high optical quality factors, and can be used to optically drive high-Q mechanical modes of the liquid-containing resonator. The methods employed here are directly applicable towards actuating optically transparent shelltype MEMS resonators for gyroscope applications. Hybrid

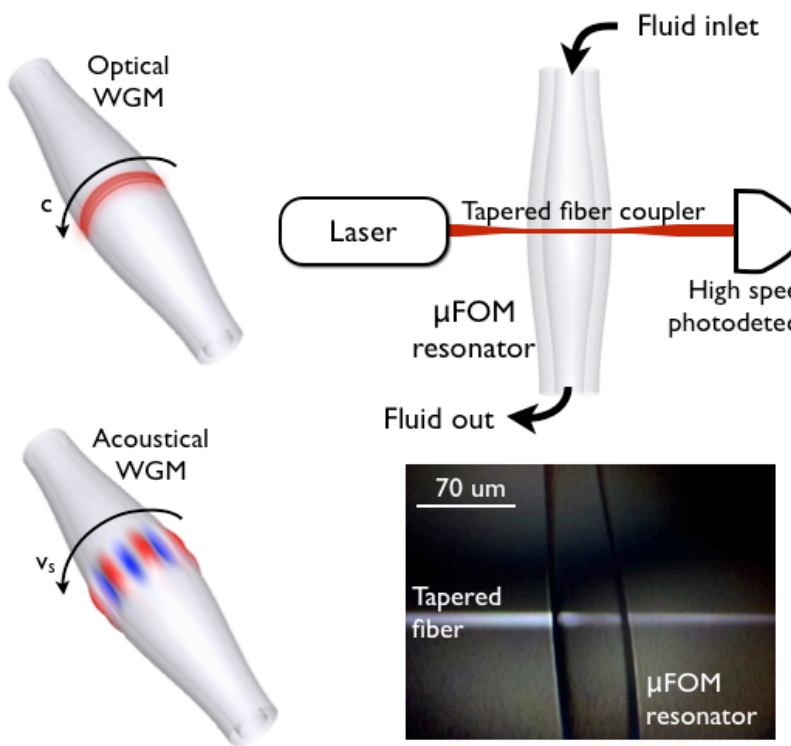

Figure 1. The optical modes and the mechanical modes of a fused silica glass $\mu F O M$ device are both whispering-gallery modes (WGM). Laser light at $1.5 \mu \mathrm{m}$ wavelength is coupled into the optical modes via a tapered optical fiber. Light then excites mechanical vibration by means of optical electrostriction. optomechanical bio/chemical sensors are also enabled.

\section{FABRICATION AND TESTING}

The $\mu \mathrm{FOM}$ device is fabricated with a commercial fused silica glass capillary that is pulled lengthwise while being heated under IR-laser illumination. The radius of the capillary is modulated in this process by varying laser power (Fig 1). The glass wall thickness is easily controlled between $5 \mu \mathrm{m}-45 \mu \mathrm{m}$ with a choice of an appropriate starting capillary. Combined with a glassblowing technique [10] the capillary wall can be as thin as $560 \mathrm{~nm}$. Such glass-based capillary devices are naturally integrated for rapid delivery of small volumes of analyte for sensing applications. We perform all the described experiments with distilled water present inside the $\mu \mathrm{FOM}$ device.

\section{EXPERIMENT}

At its widest the fused silica device forms an optical microresonator (Fig 1) that allows the 3D confinement of light due to total internal reflection. The optical modes are whispering gallery modes (WGMs) residing at the outer surface of the device. Light is coupled into these optical WGMs by means of a tapered optical fiber. Measurements indicate optical quality factors in excess of $\mathrm{Q}_{0}=10^{8}$. Mechanical oscillations in the device modulate or scatter the light in the optical WGM and are therefore observable. The minimum power observed to excite these oscillations is in approximately $160 \mu \mathrm{W}$.

The mechanical modes that we probe in this study are acoustical whispering-gallery modes [11]. Previously inaccessible using MEMS based techniques (electrostatic, thermal, piezoelectric actuation), these oscillations are excited by means of optical electrostriction as described in [6]. Mechanical modes ranging over three orders-of-magnitude in frequency are obtained (Fig 2). In addition, low frequency wineglass modes (Fig 2a) can be actuated by means of centrifugal radiation pressure. The wineglass mode and the $99 \mathrm{MHz}$ acoustical WGM (Fig 2b) are of interest as they causes significant deformation of the inner surface (Fig 3a,b), allowing strong interaction with fluids or analytes confined inside.

The mechanical quality factor $\left(\mathrm{Q}_{\mathrm{m}}\right)$ for the $99 \mathrm{MHz}$ mode is measured to be 4700 with distilled water present inside the capillary. This value compares extremely well against the previously measured $\mathrm{Q}_{\mathrm{m}}=12,300$ in a solid silica sphere (dry) for a $95 \mathrm{MHz}$ mode [8]. The displacement of the inner surface in the case of the higher frequency modes (Fig $\mathbf{2 c , d}$ ) can be improved by using thinner-walled devices [10]. Finally, the vibration can be kept stable for long periods of time, as required for sensor applications (Fig 3c).

\section{DISCUSSION}

Mechanical WGMs have a distinct advantage for sensor applications as the absence of any stationary nodes provides some level of insensitivity to position of the analyte. This is different from using standing-wave resonators or cantilever resonators [1] where sensitivity has a strong dependence on position. In addition to high- $\mathrm{Q}_{\mathrm{m}}$ mechanical WGMs, we have also observed breathing mode oscillations (typically $30 \mathrm{MHz}$ ) in these $\mu \mathrm{FOM}$ devices. 
a
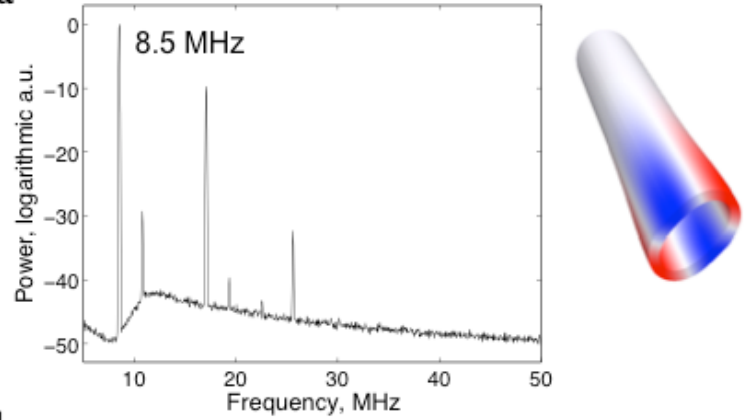

b

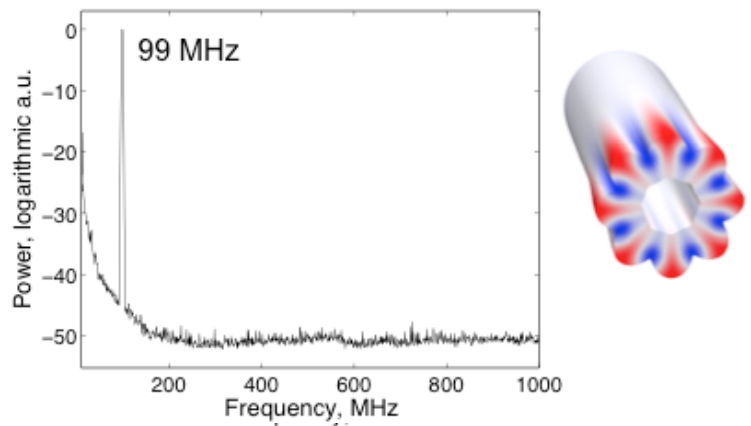

C
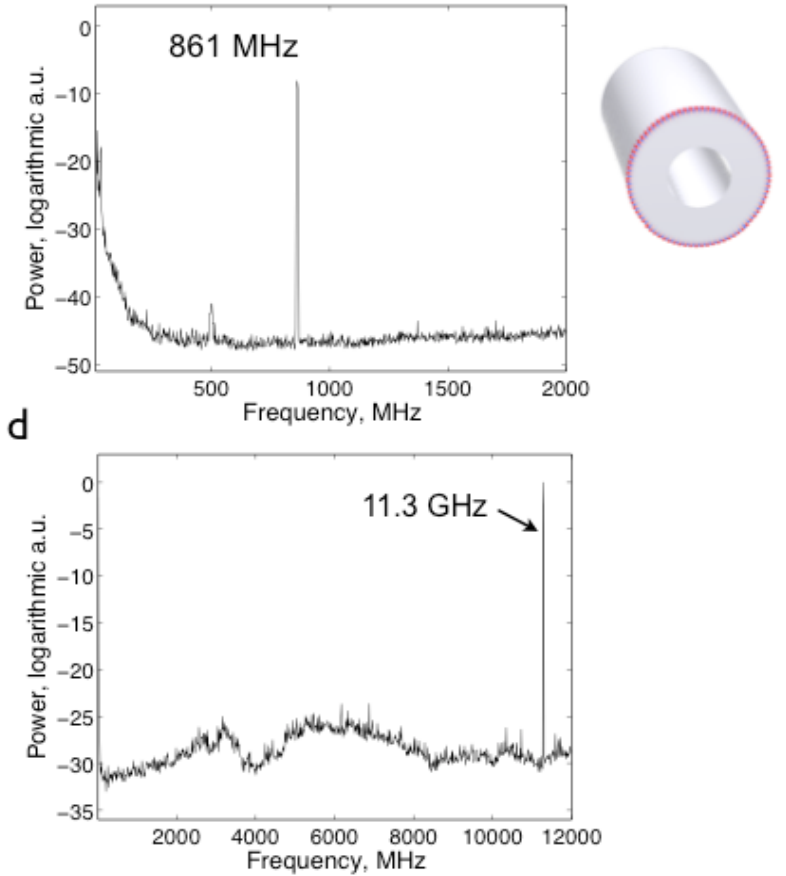

Figure 2. Mechanical vibrations ranging from $8.5 \mathrm{MHz}-11 \mathrm{GHz}$ can be excited on $\mu F O M$ devices with liquid present inside the device. The mechanical whispering gallery modes $(b, c, d)$ are traveling modes. The wineglass mode (a) is a standing wave mechanical resonance as evidenced by the harmonics present in the electrical spectrum. All these modes can interact with fluids in the device, provided the device wall thickness is comparable to the acoustical wavelength. This is easily seen for the $99 \mathrm{MHz}$ mode.

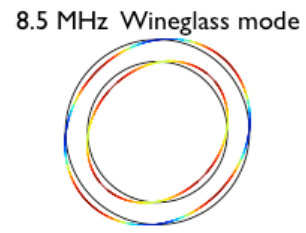

b

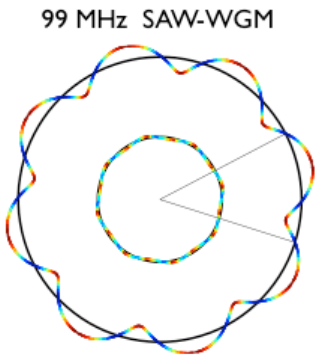

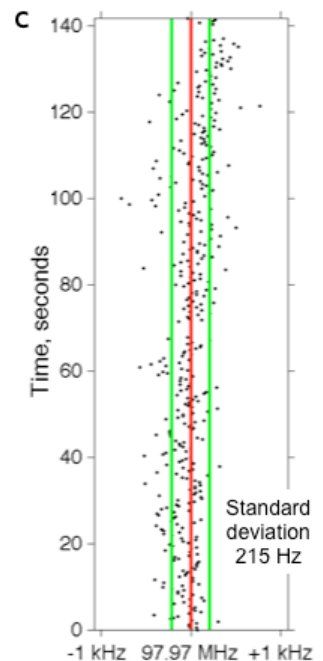

Figure 3. (a) The wineglass mode causes significant surface displacement of the inner wall of the capillary. (b) For the $99 \mathrm{MHz}$ mode, the ratio of inner surface deformation to outer surface deformation is approximately $16 \%$. (c) This oscillation exhibits good stability under constant input laser power.

\section{CONTACT}

Gaurav Bahl, bahlg@umich.edu

Tal Carmon, tcarmon@umich.edu

\section{REFERENCES}

[1] Burg, T.P., et al., "Weighing of biomolecules, single cells and single nanoparticles in fluid." Nature, 2007. 446(7139): p. 1066-1069.

[2] Vollmer, F and Arnold, S, "Whispering-gallery-mode biosensing: label-free detection down to single molecules," Nature Methods 5, 7 (2008).

[3] He, L., et al., "Detecting single viruses and nanoparticles using whispering gallery microlasers", Nature Nanotechnology, 2011. 6: p. 428-432.

[4] Eichenfield, M., et al, "A picogram- and nanometer-scale photonic-crystal optomechanical cavity," Nature 459, p. 550 (2009).

[5] Carmon, T., et al, "Temporal behavior of radiation-pressureinduced vibrations of an optical microcavity phonon mode" Physical Review Letters 94, 223902 (2005).

[6] Bahl, G., et al, "Stimulated optomechanical excitation of surface acoustic waves in a microdevice". Nature Communications 2, 403 (2011).

[7] Rokhsari, H., et al, "Radiation-pressure-driven micromechanical oscillator" Optics Express 13, 5293 (2005).

[8] Bahl, G., et al, "Observation of Spontaneous Brillouin Cooling" Nature Physics 8, 3 (2012) doi:10.1038/nphys2206.

[9] Povinelli, M. L. et al. "Evanescent-wave bonding between optical waveguides." Optics Letters 30, 3042-3044 (2005).

[10] Lee, W., et al, "A quasi-droplet optofluidic ring resonator laser using a micro-bubble.” Applied Physics Letters 99, 091102 (2011)

[11] John Zehnpfennig, et al., "Surface optomechanics: Calculating optically excited acoustical whispering gallery modes in microspheres." Optics Express, Vol. 19, pp. 14240-8 (2011). 